\title{
Acute Lymphoblastic Leukemia Following Incomplete Kawasaki Disease
}

\author{
Miguel Garcia-Dominguez ${ }^{\mathrm{a}, \mathrm{g}}$, Julio Cesar Valadez ${ }^{\mathrm{b}}$, Edgardo Tostado-Morales ${ }^{\mathrm{c}}$, \\ Jorge Luis Guzman-Rendon ${ }^{\mathrm{d}}$, Giordano Perez-Gaxiola ${ }^{\mathrm{e}}$, \\ Edna Venegas-Montoya ${ }^{\mathrm{f}}$
}

\begin{abstract}
Kawasaki disease (KD) is a multisystemic vasculitis of unknown etiology, typically affecting children younger than 5 years of age. A direct relationship between $\mathrm{KD}$ and the development of malignant tumors has not been demonstrated, however, the immunological alterations of KD could be associated with its development. An 11-month-old male was diagnosed with incomplete KD. No coronary abnormalities were detected. He was treated with intravenous immunoglobulin (IVIG) and aspirin. Four weeks later, he developed fever, otitis media, bullous pharyngitis, irritability, anemia and hyperleukocytosis, and neutropenia. Blasts forms were observed in peripheral blood. Bone marrow smear demonstrated acute lymphoblastic leukemia (ALL). $\mathrm{KD}$ has diverse clinical presentations, atypical manifestations, and several complications such as macrophage activation syndrome. As our case highlights, lymphoid neoplasms may follow KD.
\end{abstract}

Keywords: Kawasaki disease; Lymphoid neoplasms; Acute lymphoblastic leukemia

\section{Introduction}

Kawasaki disease (KD) was initially described by Tomisaku Kawasaki in 1967. It was, also called mucocutaneous lymph node syndrome. It is characterized by fever, cervical lymphad-

Manuscript submitted August 17, 2020, accepted August 28, 2020

Published online September 23, 2020

aDepartment of Clinical Immunology, Hospital Pediatrico de Sinaloa, Culiacan, Mexico

${ }^{b}$ Department of Pediatrics, Hospital Pediatrico de Sinaloa, Culiacan, Mexico 'Department of Emergency, Hospital Pediatrico de Sinaloa, Culiacan, Mexico ${ }^{\mathrm{d}}$ Department of Oncology, Hospital Pediatrico de Sinaloa, Culiacan, Mexico eEvidence-Based Medicine Department, Hospital Pediatrico de Sinaloa, $\mathrm{Cu}-$ liacan, Mexico

fDepartment of Clinical Immunology, Hospital de Especialidades, UMAE 25 IMSS, Monterrey, Mexico

gCorresponding Author: Miguel Garcia-Dominguez, Department of Clinical Immunology, Hospital Pediatrico de Sinaloa, Culiacan, Mexico.

Email: miguelgarcia.alergia@gmail.com

doi: https://doi.org/10.14740/jmc3569 enopathy, rash, non-purulent conjunctival injection, lip and mouth changes (strawberry tongue and fissured lips), and inflammation in palms and feet [1].

Although KD is the main cause of childhood acquired heart disease, its etiology is still unknown. The treatment of choice is intravenous immunoglobulin (IVIG) in one dose of 2 $\mathrm{g} / \mathrm{kg}$ and acetylsalicylic acid [2].

Different clinical presentations, severity and complications have been identified through the years since 1967, as well as the association with other pathologies such as autoimmune disorders, inborn errors of immunity and less frequently oncological diseases. Association of autoimmune diseases and cancer is well established and can develop in the course of the disease or related to immunosuppressive treatment $[3,4]$.

\section{Case Report}

An 11-month-old male diagnosed with incomplete $\mathrm{KD}$ after 4 days of fever, polymorphic rash in chest and abdomen (Fig. 1), bilateral conjunctivitis with purulent discharge and left cervical adenopathy, irritability and hyporexia.

Laboratories revealed hemoglobin $(\mathrm{Hb}) 9.5 \mathrm{~g} / \mathrm{dL}$, leukocytes $18,730 / \mathrm{mm}^{3}$, neutrophils $2,720 / \mathrm{mm}^{3}(14.5 \%)$, lymphocytes $15,300 / \mathrm{mm}^{3}$, platelets $405,000 / \mathrm{mm}^{3}$, erythrocyte sedimentation rate (ESR) $50 \mathrm{~mm} / \mathrm{h}$, C-reactive protein (CRP) 25.8



Figure 1. Polymorphic erythema predominantly in the chest and abdomen in patient with diagnosis of KD. KD: Kawasaki disease. 




Figure 2. Hypercellular bone marrow with proliferation of immature L1/ L2 lymphoblast precursor cells (arrows) with morphological classification of FAB $(90 \%)$. Sparse cytoplasm, fine chromatin with prominent nucleoli in cells. FAB: French-American-British.

$\mathrm{mg} / \mathrm{dL}$, lumbar puncture without alterations. He was started on IVIG $(2 \mathrm{~g} / \mathrm{kg})$ and aspirin $30 \mathrm{mg} / \mathrm{kg} / \mathrm{day}$ with remission of fever and inflammatory markers at $24 \mathrm{~h}$. The echocardiogram did not find coronary abnormalities.

Four weeks after the diagnosis he developed fever, daily, 3 - 4 episodes of $38.5^{\circ} \mathrm{C}$, rhinorrhea, bullous pharyngitis, right otitis media, hyporexia, vomiting and irritability. He received treatment with acyclovir and an unspecified antibiotic. Laboratories showed $\mathrm{Hb} 7.3 \mathrm{~g} / \mathrm{dL}$, leukocytes $36,890 / \mathrm{mm}^{3}$, neutrophils $730 / \mathrm{mm}^{3}(2 \%)$, lymphocytes $34,470 / \mathrm{mm}^{3}$, platelets $237,000 / \mathrm{mm}^{3}$, blasts $38 \%$, ESR $61 \mathrm{~mm} / \mathrm{h}$, CRP $4.8 \mathrm{mg} / \mathrm{dL}$, ferritin $325.67 \mathrm{ng} / \mathrm{mL}$, fibrinogen $449 \mathrm{mg} / \mathrm{dL}$, procalcitonin $0.09 \mathrm{ng} / \mathrm{dL}$, albumin $3.8 \mathrm{~g} / \mathrm{dL}$, lactate dehydrogenase (LDH) $408 \mathrm{U} / \mathrm{L}$, aspartate aminotransferase (AST) $27 \mathrm{U} / \mathrm{L}$, alanine aminotransferase (ALT) $7 \mathrm{U} / \mathrm{L}$, total bilirubin $0.16 \mathrm{mg} / \mathrm{dL}$. Because of the persistent symptoms and abnormal cell blood count a bone marrow aspiration was performed, in which $90 \%$ blasts were found (Fig. 2). He started treatment with prednisone $60 \mathrm{mg} / \mathrm{m}^{2}$ and high-risk leukemia protocol. A diagnosis of acute lymphoblastic leukemia (ALL) Pro-B, of high-risk by age, deoxyribonucleic acid (DNA) index 1.1 with 12:21 translocation was established. Cerebrospinal fluid and echocardiogram were reported normal.

\section{Discussion}

$\mathrm{KD}$ is a febrile vasculitis disorder which is usually diagnosed by a cluster of signs and symptoms along with supporting laboratory findings. It is a multisystem disorder, with predilection for small and medium sized arteries, especially coronary arteries. If left untreated, it can lead to various complications like coronary artery aneurysm, thrombosis, stenosis and even sudden death.

Diagnosis can be made with the established criteria of persistent fever for 5 days with additional at least four of the five following symptoms: bilateral conjunctival injection, changes in lips and oral mucosa, polymorphous exanthema, cervical lymphadenopathy, usually unilateral, changes in hands and feet: erythema, edema and desquamation. The American Heart Association and the American Academy of Pediatrics recommend a combination of aspirin and IVIG for treating acute $\mathrm{KD}$.

Autoimmune diseases have been associated with the presence of solid or hematological malignancies [3]. The levels of soluble interleukin 2 (IL-2), tumor necrosis factor (TNF), cluster of differentiation 4 (CD4), CD8, arachidonic acid, free radicals, accelerated proliferation of differentiated fibroblasts in regions of repeated epithelial aggression, as well as viral infections, epigenetic changes, immunosuppression and DNA damage induce cell proliferation, apoptosis resistance, angiogenesis, transformation and mutagenesis [5-8].

Yu et al reported a cohort of 75,123 patients with autoimmune diseases in Taiwan from 1997 to 2012, where 3.78\% developed cancer [9]. Rheumatoid arthritis was the largest group with $46.8 \%$, systemic lupus erythematosus $27.8 \%$, Sjogren's syndrome $15.9 \%$, and ectopic kidney (EK), which represented $4.61 \%$. They found a higher overall risk for women and ages younger than 20 years. Of the 3,469 patients with $\mathrm{KD}$, the average age was 3 years, 11 patients $(0.31 \%)$ developed cancer, with a female/male ratio of 0.56 and an incidence of $34.7 / 100,000$ people. The 11 patients were under 20 years old and were diagnosed with non-Hodgkin lymphoma in five patients, bone tumors and soft tissue sarcomas in four, and leukemias in two of them. The highest risk of developing cancer in autoimmune diseases including KD was during the first year of follow-up, they were predominantly males, with a time of onset of malignancy between 4 weeks and 24 years [9].

The literature describes cases of acute leukemia that occur after KD. Murray et al reported a 3-year-old boy with complete $\mathrm{KD}$, he was treated with IVIG and aspirin, however 10 weeks later he developed bruises, paleness and hepatosplenomegaly. Bone marrow examination demonstrated for ALL [10]. Suzuki et al reported a 2-year-old boy with complete $\mathrm{KD}$, he was treated with IVIG and aspirin. Seven weeks later he developed petechiae in lower extremities and hepatosplenomegaly. Examination of the bone marrow demonstrated of ALL of the high risk [11]. Nakamura et al reported a child who died 3 weeks after the acute phase of $\mathrm{KD}$, the cause of death being acute leukemia $[12,13]$. Recently Lee et al reported an 11-monthold male with incomplete KD with mildly-dilated coronary arteries. He was treated with IVIG. He presented anemia and recurred 2 weeks later with leukocytosis, a bone marrow examination demonstrated acute megakaryocytic leukemia [14]. In a patient with monocytic leukemia, KD-like was diagnosed 1 week after the start of chemotherapy, with an echocardiogram showing the presence of ectasia of the left coronary artery. He was treated with IVIG and steroids with remission of symptoms [15].

\section{Conclusions}

$\mathrm{KD}$ is a vasculitis where processes of inflammation and autoimmunity intervene. Association with oncological disease has 
been shown predominantly in male children. Therefore, it is important in a patient who has criteria for KD, which does not improve with the usual treatment or starts with atypical behavior of the disease or alterations in the cell count, consider a timely diagnostic approach to concomitant oncological diseases to improve the life expectancy of the patient.

\section{Acknowledgments}

None to declare.

\section{Financial Disclosure}

None to declare.

\section{Conflict of Interest}

None to declare.

\section{Informed Consent}

Informed consent was obtained.

\section{Author Contributions}

$\mathrm{MG}$, JV, and JG were the treating physicians and the monitoring of the hematological disease. EV wrote the manuscript with the support of ET, MG and GP supervised the project. All authors discussed the results and contributed to the writing of the final manuscript.

\section{Data Availability}

Any inquiries regarding supporting data availability of this study should be directed to the corresponding author.

\section{References}

1. Kawasaki T. [Acute febrile mucocutaneous syndrome with lymphoid involvement with specific desquamation of the fingers and toes in children]. Arerugi. 1967;16(3):178222.

2. McCrindle BW, Rowley AH, Newburger JW, Burns JC, Bolger AF, Gewitz M, Baker AL, et al. Diagnosis, treatment, and long-term management of Kawasaki disease: a scientific statement for health professionals from the American Heart Association. Circulation. 2017;135(17):e927-e999.

3. Bernatsky S, Ramsey-Goldman R, Clarke A. Malignancy and autoimmunity. Curr Opin Rheumatol. 2006;18(2):129-134.

4. Pasquet F, Pavic M, Ninet J, Hot A. [Autoimmune diseases and cancers. Part I: cancers complicating autoimmune diseases and their treatment]. Rev Med Interne. 2014;35(5):310-316.

5. Turesson C, Matteson EL. Malignancy as a comorbidity in rheumatic diseases. Rheumatology (Oxford). 2013;52(1):5-14

6. Okada F. Inflammation-related carcinogenesis: current findings in epidemiological trends, causes and mechanisms. Yonago Acta Med. 2014;57(2):65-72.

7. Trinchieri G. Cancer and inflammation: an old intuition with rapidly evolving new concepts. Annu Rev Immunol. 2012;30:677-706.

8. Franks AL, Slansky JE. Multiple associations between a broad spectrum of autoimmune diseases, chronic inflammatory diseases and cancer. Anticancer Res. 2012;32(4):1119-1136.

9. Yu KH, Kuo CF, Huang LH, Huang WK, See LC. Cancer risk in patients with inflammatory systemic autoimmune rheumatic diseases: a nationwide population-based dynamic cohort study in Taiwan. Medicine (Baltimore). 2016;95(18):e3540.

10. Murray JC, Bomgaars LR, Carcamo B, Mahoney DH, Jr. Lymphoid malignancies following Kawasaki disease. Am J Hematol. 1995;50(4):299-300.

11. Suzuki H, Takeuchi T, Minami T, Shima Y, Iwahashi S, Shibuta S, Uemura S, et al. Neoplasms in three patients following Kawasaki disease. Pediatr Int. 2005;47(2):217219.

12. Nakamura Y, Aso E, Yashiro M, Uehara R, Watanabe M, Tajimi M, Oki I, et al. Mortality among persons with a history of Kawasaki disease in Japan: can paediatricians safely discontinue follow-up of children with a history of the disease but without cardiac sequelae? Acta Paediatr. 2005;94(4):429-434.

13. Nakamura Y, Aso E, Yashiro M, Tsuboi S, Kojo T, Aoyama Y, Kotani K, et al. Mortality among Japanese with a history of Kawasaki disease: results at the end of 2009. J Epidemiol. 2013;23(6):429-434.

14. Lee B, Kofman CD, Tremoulet AH, Kuo DJ. Fever without a source: evolution of a diagnosis from Kawasaki disease to acute myelogenous leukaemia. BMJ Case Rep. 2017;2017.

15. Akita H, Matsuoka S, Takahashi Y, Watanabe T, Takaue Y, Kuroda Y. Kawasaki-like disease in early course of acute monocytic leukaemia. Eur J Pediatr. 1992;151(3):177178. 